\title{
AN ABSORPTION BAND IN ETHYLENE GAS IN THE NEAR INFRARED
}

\author{
By Richard M. Badger and John L. Binder \\ Gates Chemical Laboratory, California Institute of Technology
}

(Received August 12, 1931)

ABSTRACT

The absorption spectra of gaseous ethane and ethylene have been investigated by photographic methods in the region $\lambda \lambda 6500-9500$. In the case of ethane no absorption was found. In ethylene a rather strong band was found at $\lambda 8720$ which has a structure resembling that predicted by $\mathrm{H}$. H. Nielsen for the oscillation-rotation bands of a molecule with moments of inertia in the ratio $1.4: 1: 0.14$. The following very provisional values for the moments of inertia of the ethylene molecule are given: $A_{x}=31 \times 10^{-40}$, $A_{y}=27 \times 10^{-40}, A_{z}=3.8 \times 10^{-40}$.

\section{INTRODUCTION}

$I^{\mathrm{N}}$

$\mathrm{N}$ THE cases of some special polyatomic molecules where two of the moments are equal, either due to the symmetry of the molecule, as in ammonia, ${ }^{1}$ or to a linear structure, as in acetylene ${ }^{2}$ and hydrogen cyanide, ${ }^{3}$ the vibration rotation spectra have submitted to a satisfactory analysis. But for the general case of a molecule with three unequal moments of inertia we have as yet no example which has been subjected to anything like a thorough treatment. In fact the spectrum of what might be expected to be a very simple case, the water molecule, has resisted many attempts at analysis.

One might expect the problem to be somewhat simplified in the case of a molecule which approximates a symmetrical rotator, in that two of the moments of inertia are nearly equal. A rather simple example of a molecule of this type is ethylene. The infrared spectrum of ethylene has been thoroughly investigated by Levin and Meyer ${ }^{4}$ with thermocouple technique, but the resolving power employed was not quite sufficient to allow an analysis of the bands. Some time ago, during some preliminary work in the photographic infrared, an ethylene band was observed by one of the authors, ${ }^{5}$ which was comparatively well resolved and which showed some interesting features in its structure. The present article describes further work, in which considerable improvement in the resolution of the band has been obtained.

\section{Experimental Procedure}

The ethylene gas used in this investigation was a commercial product prepared for anaesthetic purposes, guaranteed to be 99.2 percent pure. It was confined in a steel tube $280 \mathrm{~cm}$ in length, closed at one end with a plate glass

${ }^{1}$ R. M. Badger and R. Mecke, Zeits. f. phys. Chem. Abt. B, 5, 333 (1929).

${ }^{2}$ K. Hedfeld and R. Mecke, Zeits. f. Physik. 64, 151 (1930).

${ }^{3}$ R. M. Badger and J. L. Binder, Phys. Rev. 37, 800 (1931).

${ }^{4}$ A. Levin and C. F. Meyer, J. Opt. Soc. Am. 16, 137 (1928).

${ }^{5}$ R. M. Badger, Phys. Rev. 35, 1433 (1930). 
window and at the other with a mirror. The light from the tungsten lamp source traversed the tube twice before entering the slit of the spectrograph. The latter contained a ten-foot grating in an Eagle mounting. The exposures were made in the first order using the iron arc spectrum in the second order for calibration. In most of the experiments the gas pressure employed was three atmospheres, in a few others about half an atmosphere. Eastman infrared sensitive plates hypersensitized in ammonia were used.

Since the absorption band observed is very complex, and since the resolving power of the photographic plate for absorption spectra is much less than for emission spectra, especially when the absorption is weak and the contrast poor, the following method was used in working up the results. A number of plates were exposed and from these the four best ones were chosen, and were enlarged about four times on fine-grained contrast plates. From these, horizontal regions free from pinholes and other blemishes were chosen and enlargements made on contrast film, using a weak cylindrical lens together with the camera lens to introduce astigmatism and get rid of grain. The four films which resulted were carefully superposed, and a reduced copy of this composite picture was made and photometered.

One has to be much more careful in introducing astigmatism to get rid of grain in absorption spectra than in emission spectra, since pin holes in the continuous background are likely to be mistaken for absorption lines. We believe that this difficulty has been avoided and the method worked very well as lines which were very doubtful on the original negatives came out quite definitely in the final picture. As can be seen on the photometer curve reproduced, the effect of grain has been almost eliminated and it is probable that even the small humps on the curve are significant.

In each of the several photographic procedures the contrast in the regions of weak absorption was improved, but with a corresponding loss of detail in the region of strong absorption, as can be seen on the photometer curve. The weak lines were consequently measured on the photometer curve, and the strong lines on another composite picture with less contrast, made from two other of the original negatives.

\section{ExPERIMENTAL RESUlts}

A careful investigation of the region $\lambda \lambda 6500-9500$ resulted in the discovery of only one absorption region in ethylene gas. This is a very complex band, near the center of which are two strong maxima about 8 Angstrom units apart, which have the appearance of the $Q$ branches found in the simpler oscillationrotation bands. The absorption on the short wave-length side of these maxima seems to be greater than on the opposite side, but this may be due to the fact that this side of the band is better resolved. The band does not have very much resemblance to any of the ethylene bands found by Levin and Meyer ${ }^{4}$ in the farther infrared. However, if the spacing between groups of closely neighboring lines is compared with the spacing of the maxima of the latter bands, the band at $\lambda 8720$ is somewhat similar to the band at $3.2 \mu$ on the short wave-length side; and to the band at $2.3 \mu$ on the long wave-length side. 
TABLE I, The ethylene band at $\lambda 8720$.

\begin{tabular}{|c|c|c|c|c|c|}
\hline line & $\lambda$ & $\nu\left(\mathrm{cm}^{-1}\right)$ & line & $\lambda$ & $\nu\left(\mathrm{cm}^{-1}\right)$ \\
\hline+14 & $\left\{\begin{array}{r}8672.0 \\
74.3\end{array}\right.$ & $\begin{array}{r}11528.2 \\
25.2\end{array}$ & \multirow[t]{2}{*}{0} & $\left\{\begin{array}{r}8719.9 \\
20.4\end{array}\right.$ & $\begin{array}{r}11464.8 \\
64.2\end{array}$ \\
\hline+13 & $\left\{\begin{array}{l}77.2 \\
78.6\end{array}\right.$ & $\begin{array}{l}21.3 \\
19.4\end{array}$ & & 22.1 & 61.9 \\
\hline+12 & $\left\{\begin{array}{l}79.9 \\
80.7 \\
81.7 \\
82.4\end{array}\right.$ & $\begin{array}{l}17.6 \\
16.7 \\
15.3 \\
14.4\end{array}$ & \multirow[t]{2}{*}{$\begin{array}{l}-1 \\
-2 \\
-3\end{array}$} & $\begin{array}{r}\left\{\begin{array}{l}23.6 \\
24.5\end{array}\right. \\
26.6\end{array}$ & $\begin{array}{l}60.0 \\
58.8 \\
56.1\end{array}$ \\
\hline+11 & $\left\{\begin{array}{l}84.9 \\
86.5\end{array}\right.$ & $\begin{array}{l}11.0 \\
08.9\end{array}$ & & 29.9 & 51.7 \\
\hline+10 & 91.7 & 02.1 & & 32.3 & 48.6 \\
\hline+9 & 93.2 & 00.1 & \multirow{2}{*}{-4} & $\left\{\begin{array}{l}33.9 \\
34.9\end{array}\right.$ & $\begin{array}{l}46.5 \\
45.2\end{array}$ \\
\hline+8 & $\left\{\begin{array}{l}94.8 \\
95.8\end{array}\right.$ & $\begin{array}{r}11498.0 \\
96.7\end{array}$ & & 36.5 & 43.0 \\
\hline+7 & 870.33 & 87.2 & -5 & $\left\{\begin{array}{l}39.9 \\
41.2\end{array}\right.$ & $\begin{array}{l}38.6 \\
37.0\end{array}$ \\
\hline+6 & 05.0 & 84.6 & \multirow[t]{2}{*}{-6} & $\left\{\begin{array}{l}44.2 \\
45.3\end{array}\right.$ & $\begin{array}{l}33.0 \\
31.5\end{array}$ \\
\hline+5 & 07.8 & 80.9 & & $(47.9$ & 28.1 \\
\hline+4 & $\begin{array}{l}09.3 \\
10.5\end{array}$ & $\begin{array}{l}78.8 \\
77.2\end{array}$ & -7 & $\left\{\begin{array}{l}50.1 \\
51.0\end{array}\right.$ & $\begin{array}{l}25.2 \\
24.0\end{array}$ \\
\hline+3 & 12.2 & 75.0 & \multirow[t]{4}{*}{-8} & \multirow[t]{4}{*}{$\begin{array}{l}54.6 \\
55.9\end{array}$} & \multirow[t]{4}{*}{$\begin{array}{l}19.4 \\
17.8\end{array}$} \\
\hline+2 & 13.4 & 73.4 & & & \\
\hline+1 & $\left\{\begin{array}{l}15.2 \\
16.4\end{array}\right.$ & $\begin{array}{l}71.0 \\
69.4\end{array}$ & & & \\
\hline & 18.1 & 67.2 & & & \\
\hline
\end{tabular}

Brackets indicate definite regions of strong absorption which may, however, have a more complex structure than that indicated by the maxima given. The lines which are not numbered are doubtful.

The wave-length and frequency of absorption maxima, measured in the way described above under experimental procedure will be found in Table I, and a graphical representation of the band, together with a reproduction of a microphotometer curve, in Fig. 1. It is believed that all the maxima recorded are real with a few possible exceptions which are indicated in the table.

It should be mentioned at this point that ethane gas was also investigated in the same region as ethylene, but at pressures up to one atmosphere, in the absorption tube described above, no absorption was found. This is unfortunate since ethane has some points of special interest, especially the possibility of free rotation of the methyl groups relative to each other, about the carbon-carbon bond. However, in this investigation it was not possible to obtain enough pure gas to work at appreciably higher pressures. Ethane prepared from natural gas was found to contain methane in disturbing amounts, which could not be completely removed, and the gas used had to be prepared from ethylene bromide with the use of a zinc-copper couple. 


\section{Discussion of Results}

The results of this investigation are here presented, though their complete analysis is as yet lacking, because of the rather striking similarity between the spectrum observed and that which has been predicted by quantum mechanics. The theory of the unsymmetrical rotator was worked out some time ago, but it is only recently that the results were put in a form such that they might readily be compared with experiment. In the last few months D. M. Dennison ${ }^{6}$ and H. H. Nielsen ${ }^{7}$ have calculated the vibration rotation band structure to be expected from plane, unsymmetrical rotators having moments of inertia in various ratios, and these results have beeen presented in diagrams which can readily be compared with observed spectra. In the present problem the diagrams of Nielsen are more useful, and copies of these were very kindly sent to the authors in advance of their publication.

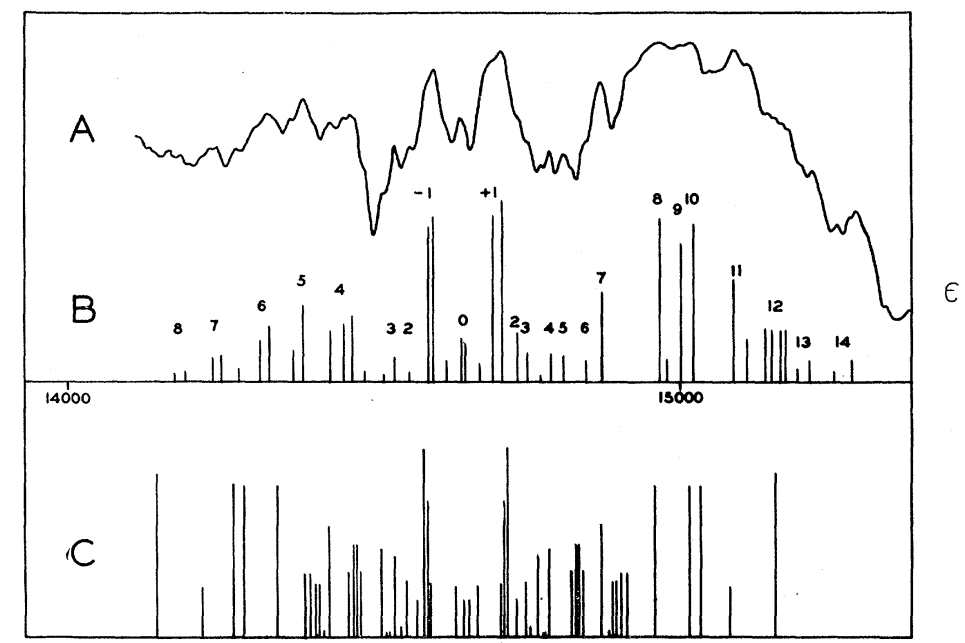

Fig. 1. The ethylene band at $\lambda 8720 . A$, Photometer curve of a composite picture made from four negatives. $B$, The principal maxima plotted on a frequency scale. $C$, Predicted spectrum for a molecule with constant moments of inertia in the ratio $1.14: 1: 0.14$, when the change of electric moment due to vibration is along the intermediate axis. (Calculated by H. H. Nielsen).

The predicted spectra were necessarily calculated for a very much idealized case. A rigid molecule was assumed, the effect of special symmetry properties of the molecule on the intensities of the lines was not considered, and the Boltzmann factor was neglected. However, in Fig. 1, in which the observed ethylene band at $\lambda 8720$ is compared with the spectrum predicted for an ideal molecule with moments of inertia in the ratio $1.14: 1: 0.14$, a considerable similarity is evident. In particular one notes the two strong absorption regions which have the appearance of $Q$ branches, and several of the more intense lines on the high frequency side of the band. On the low frequency side the similarity is less evident. One might of course expect a marked dissymmetry in the band due to the effect of symmetry properties, and due to the stretching of the molecule as it vibrates. The second effect is difficult to

${ }^{6}$ D. M. Dennison, Reviews of Modern Physics 3, 280 (1931).

${ }^{7}$ H. H. Nielsen, Phys. Rev. 38, 1432 (1931). 
take into account at present since it is not quite certain what kind of oscillation gives rise to the band in question. It is rather probable that it is an overtone of a very active nonharmonic fundamental oscillation, since it is the only band observed in the photographic region. If so, it is probably the fourth harmonic of the fundamental at $3107 \mathrm{~cm}^{-1}$, but the possibility is not entirely excluded that it may be a combination band. Further work in the intermediate infrared will probably be necessary to decide this point definitely.

If the correspondence between the observed ethylene band the predicted spectrum is real, it is possible from Nielsen's diagrams to estimate approximate moments of inertia of the ethylene molecule. These are $A_{x}=31 \times 10^{-40}$, $A_{y}=27 . \times 10^{-40}$, and $A_{z}=3.8 \times 10^{-40}$. These values are given provisionally. If one assumes that the angle between the bonds joining two hydrogen atoms to one carbon atom is the same as in methane, $109^{\circ} 28^{\prime}$, one finds for the $\mathrm{C}-\mathrm{C}$ separation $1.32 \mathrm{~A}$, and for the $\mathrm{C}-\mathrm{H}$ separation $0.92 \mathrm{~A}$, which are certainly quite reasonable values.

Note added August 31, 1931. The authors have recently received from H. H. Nielsen copies of some of the new diagrams of predicted types of oscillation-rotation bands which appear in an article by him in this issue of the Physical Review. ${ }^{7}$ These figures are more complete than the preliminary ones which were available at the time of writing of this article in that they include additional lines due to quantum levels for which $J$ has the values 5 and 6 , and that a correction has been made to the intensities which takes account of the Boltzmann factor. These changes slightly alter the general aspect of the figures and suggest a somewhat different interpretation of the ethylene band than that indicated above.

First of all the agreement between the experimental photometer curve and the predicted band now seems to be better for the " $C$ " type of band; that is a band due to a vibration in which the change in electric moment is normal to the plane of the molecule. This is interesting since it indicates that the band under discussion is due to a combination of one of the deformation oscillations with a harmonic of one of the so-called valence frequencies. Further, a slight change in scale in comparing photometer curve and diagram seems desirable, which of course produces a corresponding change in the estimated moments of inertia. As before the maxima designated as +1 and -1 are to be interpreted as due to the first densely packed sets of lines on the two sides of the band origin, but it would appear that the maximum +7 (and probably -4) should be correlated with the second densely packed set of lines instead of with a single line.

If one draws from the diagrams a "predicted" photometer curve, by introducing factors to take account of the finite resolving power of the spectrometer and photographic plate, one finds a much greater correspondence between the observed and predicted curves than might be expected when one considers the possible influence of symmetry factors. The agreement is indeed very good in the central portion of the band. The outer portions of the calculated diagrams are less complete and it may be necessary to calculate additional lines for values of $J$ up to about 10 before the final interpretation of the ethylene spectrum is made.

The authors are indebted to H. H. Nielsen for several helpful suggestions. 